Br Heart J 1988;59:395-6

Correspondence

\title{
Haemodynamic observations during percutaneous transluminal coronary angioplasty in the presence of synchronised diastolic coronary sinus retroperfusion
}

Sir,

We should like to comment on the important study of Beatt et al (1988);59:159-67. The development of new intracoronary interventions to protect jeopardised myocardium is a goal we share with Beatt $e t$ al. The Rotterdam team used a similar protocol and similar criteria for evaluating the possible beneficial effects of synchronised coronary sinus retroperfusion to the ones that we are currently using. We agree with their criticism of the equipment that they used and we would like to draw attention to the improved techniques of retroperfusion and retroinfusion developed during the past two years. ${ }^{1}$

They report that the method of retroperfusion that they used did not produce any convincing clinical benefit during acute myocardial ischaemia. It seems that the retroperfusion delivery equipment that they used is essentially the same system (Model EC-1) that we helped to develop but abandoned three years ago.

Beatt et al question the discrepancy between reports of obvious benefits in animal studies and those of failure in human studies. They do not indicate the weight and size of their patients but we assume that they were several times heavier than the dogs used in the animal studies. The limited volume of blood that was pumped retrogradely into the coronary veins might have been adequate in dogs but was probably insufficient in human beings. The autoinflatable balloon has a considerable negative effect on the net flow because blood is needed to fill the balloon on each pump cycle. The volume of the $10 \mathrm{~mm}$ balloon reportedly used in their study probably reduced net flow by at least $40-70 \mathrm{ml} / \mathrm{min}$. Nevertheless, in a larger study, investigators from the University of Massachusetts reported favourable results with the same system. ${ }^{2}$

We are currently using equipment manufactured by Retroperfusion Systems, Inc (RSI) of Costa Mesa, California, which incorporates many major improvements in design that should overcome the deficiencies referred to in the Rotterdam article. The dual lumen $8 \mathrm{~F}$ catheter that we are currently using has an infusion lumen with a cross sectional area greater than twice the reported cross sectional area $(56 \mathrm{~mm}$ ) of the catheter described in the Rotterdam study. The second lumen of the new catheter monitors coronary venous pressure. This catheter system can also deliver significantly greater flows because it is designed to prevent wasteful regurgitation of oxygenated blood during the pump filling stroke (down stroke).

Moreover, unpublished studies of the RSI system in 12 patients in Rouen, France, and eight patients in Los Angeles, California, yielded positive results when retroperfusion was used to support the ischaemic myocardium during percutaneous transluminal coronary angioplasty (with left ventricular function measured by cross sectional echocardiography as the major end point for efficacy).

One patient in the Los Angeles series developed sudden akinesis of the left ventricular septum and anterolateral wall, resulting in cardiogenic shock, when acute coronary artery dissection occurred during percutaneous transluminal coronary angioplasty. Retroperfusion was started immediately and within seconds the intense anginal pain vanished. Within a few minutes left ventricular function improved, blood pressure increased, and the patient was considered sufficiently stable to permit attempts to reopen the occluded artery with retroperfusion support. Retroperfusion was maintained for two hours and 30 minutes until emergency bypass surgery could be performed. The patient had an uneventful recovery and within a week was discharged without significant wall motion abnormality.

We feel we must answer the remark of the Rotterdam group who state that with retroperfusion "delivery of thrombolytic and antiarrhythmic agents is unlikely to be substantially more effective than intravenous administration." Many animal and some human studies have shown that the coronary venous 
channel can effectively deliver cardioactive agents even when the coronary arteries are occluded. ${ }^{3-7}$ Moreover, high myocardial drug concentrations, up to 100 times that measured in the normal myocardium, were obtained with retroinfusion of the coronary venous channels with dosages much lower than those normally required for systemic infusion. Therefore, we believe that retroinfusion will be an important additional technique in clinical cardiology.

Eliot Corday,

Cedars-Sinai Medical Center,

Los Angeles,

California, USA.

Jean Farcot,

Hospital Ambroise Paré,

Paris,

France.

Kevin Drury,

Cedars-Sinai Medical Center,

Los Angeles,

California, USA.

Jacques Berland,

Hospital Charles Nicolle,

Rouen,

France.

\section{References}

1 Drury JK, Yamazaki S, Fishbein MC, Meerbaum $S \overrightarrow{\bar{F}}$ Corday E. Synchronized diastolic coronary venous retroperfusion: results of a pre-clinical safety ancक efficacy study. J Am Coll Cardiol 1985;6:328-35.

2 Gore JM, Weiner BH, Benotti JR. Preliminaryo experience with synchronized coronary sinus retroperfusion in humans. Circulation 1986;74:381-8ळ

3 Karagueuzian HS, Ohta M, Drury JK, et al. Coronary venous retroinfusion of procainamide: a newi approach for the management of spontaneous and inducible sustained ventricular tachycardia during?

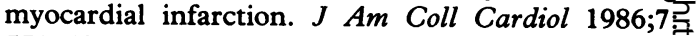
$551-63$.

4 Otsu F, Carew TW, Maroko TR. Myocardial concen 6 tration and antiarrhythmic effect of lidocaine admin $\dot{\omega}$ istered via coronary veins [Abstract]. J Am Cole Cardiol 1985;5:467.

5 Corday E, Meerbaum S, Drury JK. The coronary sinus? an alternate channel for administration of arteria $\bar{Z}$ blood and pharmacologic agents for protection andu treatment of acute cardiac ischemia. $\mathrm{J} \mathrm{Am} \mathrm{Col} \overline{\mathrm{B}}$ Cardiol 1986;7:711-4.

6 Hatori N, Miyazaki A, Drury JK, et al. Beneficial effectso of coronary venous retroinfusion of superoxide dis $-\infty$ mutase and catalase on reperfusion arrhythmias, myocardial function and infarct size in dogs [Abs tract]. Circulation 1986;74(suppl II):346.

7 Miyazaki A, Drury JK, Hatori N, Corday E. Improved clot lysis and infarct size reduction with coronaryo venous compared with intravenous infusion of streptokinase [Abstract]. J Am Coll Cardiol 1987; 9:82A.

\section{Notices}

\section{British Cardiac Society}

The Annual General Meeting for 1988 will take place in Belfast on 23 and 24 March 1988, and the closing date for receipt of abstracts was 4 January 1988 .

\section{Cardiology-Moroccan, French, American meeting}

An International Congress of Cardiology, co-spon- sored by the Council on Clinical Cardiology of the American Heart Association, will be held ing Marrakesh on 2 to 5 November 1988. Furthe information from: Dr M Benomar, Ligue Nationale de Lutte Contre les Maladies Cardiovasculaires, $B$ 1326, Rabat R P, Morocco; Dr Y Grosgogeat, Groupe Hospitalier Pitié-Salpétrière, Service dS్ Cardiologie, 47 Boulevard de l'Hôpital, 75013 Pariș France; Dr W C Sheldon, The Cleveland Clinie Foundation, Department of Cardiology, 9500 Euclid Avenue, Cleveland, Ohio 44106, USA. 